\title{
Connection between Slab and Cluster Models for Crystalline Surfaces
}

\author{
(C) R.A. Evarestov*, Th. Bredow, K. Jug \\ Universität Hannover, \\ 30167 Hannover, Germany \\ * St. Petersburg State University, \\ 198904 Stary Peterhof, St. Petersburg, Russia \\ E-mail: robert@hq.pu.ru
}

(Received 25 January 2001 г.)

\begin{abstract}
Different models for the theoretical description of bare crystalline surfaces are compared and discussed in terms of stoichiometry and conservation of the point symmetry. While infinite models such as the semi-infinite model or the slab model in general preserve the symmetry of the perfect crystal surface, special care has to be taken when finite cluster models are considered. The connection between molecular cluster choice and surface unit cell of the slab model is demonstrated for metal oxides such as $\mathrm{MgO}, \mathrm{TiO}_{2}, \mathrm{~V}_{2} \mathrm{O}_{5}$, and $\mathrm{Al}_{2} \mathrm{O}_{3}$, analyzing how atoms of the primitive unit cell of the parent three-dimensional crystal are distributed in different planes of slab and cluster models. General rules for the construction of finite cluster models based on stoichiometry and symmetry considerations are given and illustrated with calculations on water adsorption at rutile (110).
\end{abstract}

Crystalline surfaces are of high importance in many chemical and physical processes $[1,2]$. They are therefore subject of an increasing number of studies, both experimental and theoretical [3]. In the last decades, quantum-chemical calculations have become an important tool for investigations of structural, electronic and catalytic properties of surfaces. Methodological developments and the rapid improvement of computer hardware enabled theorists to treat systems of increasing complexity. Thereby it was possible not only to reproduce experimental findings with increasing accuracy, but also to aid in the interpretation of experimental results.

For simulations of the electronic structure of surfaces of crystalline solids three basic approaches are used: cluster, slab, and semi-infinite crystal models [4]. The latter is the most appropriate, because it takes into account an infinite number of atoms of the crystal below the surface [5]. Slab and cluster models are nevertheless by far more popular, since they are more feasible from the computational point of view. The cyclic cluster model [4] is intermediate between slab and molecular cluster models. It takes into account the translational symmetry of the surface but considers only a finite number of interatomic interactions within a strictly defined region.

The choice of the cluster model (both molecular and cyclic) for a surface allow to employ all those quantum chemical techniques that have been developed for molecular systems. It is also possible to study defects or adsorption reactions in the limit of low coverage. Care has to be taken in the selection of cluster size and shape due to the unavoidable presence of boundary effects. Different schemes have been developed to reduce boundary effects in the cluster model, either by embedding procedures or by the introduction of cyclic boundary conditions. Slab models eliminate twodimensional boundary effects and are widely used for the study of periodic surface structures.

In the present work, the connection between slab and cluster models based on a symmetry analysis of the crystalline surface is considered. As far as we know, this connection has not been investigated explicitly before, although it is implied in many surface studies using molecular clusters as a model.

For some metal oxides it is compared how atoms in the primitive unit cell of the three-dimensional crystal are distributed in different atomic planes of slab models and clusters. General rules for the construction of molecular cluster models for surface simultanions based on considerations of stoichiometry and symmetry are suggested.

In section 1, infinite models of surfaces, the semi-infinite crystal model and the slab model, are briefly discussed. The relationship between unit cells of two-dimensional $(2 D)$ slab models and the three-dimensional $(3 D)$ bulk unit cell is investigated. Section 2 gives a description of finite models, the cyclic cluster and the molecular cluster model, and their connection with the slab model. The results of calculations of molecular clusters with the same stoichiometry but different arrangements of atoms are compared for the adsorption behavior towards water at rutile(110).

\section{Infinite Models of Surfaces}

1.1. Semi-Infinite Crystal Model. The symmetry group of the semi-infinite crystal model contains, in addition to translations in the surface plane, only the rotations and mirror reflections which keep the atoms in the planes parallel to the surface.

The model of a semi-infinite crystal turns out to be very difficult to realize in calculations of real systems. A simplification of this model could take into account a finite number of interacting layers parallel to the surface. In this case, the $2 D$ translation symmetry in the surface plane is maintained. This simplified model is known as the slab model of the surface [6].

1.2. Slab Model. The symmetry group of a slab of finite thickness allows to perform symmetry operations that move the atoms out of the plane of the surface and bring them into equivalent positions. These are space diperiodic groups $D G$ in three dimensions (layer groups). 
Table 1. Distribution of the Bulk Primitive Unit Cell Atoms over Planes in the One-Layer Slab Model of Surfaces

\begin{tabular}{|c|c|c|c|c|}
\hline $\begin{array}{l}\text { Labeling of Atoms } \\
\text { in the Bulk Unit Cell }\end{array}$ & Surface & Plane Lattice & $\begin{array}{l}\text { Atomic Distribution } \\
\text { in Planes }\end{array}$ & Symmetry Group \\
\hline $\begin{array}{l}\mathrm{MgO} \\
\text { (primitive) } \\
1-\mathrm{Mg}, 2-\mathrm{O}\end{array}$ & $(001)$ & Square & (1) 1,2 & $D G 61-P 4 / m m m\left(D_{4 h}^{1}-G 123\right)$ \\
\hline $\begin{array}{l}\mathrm{MgO} \\
\text { (conventional) } \\
1-4-\mathrm{Mg} \\
5-8-\mathrm{O}\end{array}$ & $(001)$ & Square & $\begin{array}{l}\text { (1) } 1,2,5,6 \\
\text { (2) } 3,4,7,8\end{array}$ & $D G 61-P 4 / m m m\left(D_{4 h}^{1}-G 123\right)$ \\
\hline $\begin{array}{l}\mathrm{TiO}_{2} \\
\text { (rutile) } \\
1,2-\mathrm{Ti} \\
3-6-\mathrm{O}\end{array}$ & $(110)$ & Rectangular & $\begin{array}{l}\text { (1) } 3 \\
\text { (2) } 1,2,5,6 \\
\text { (3) } 4\end{array}$ & $D G 37-P m m m\left(D_{2 h}^{1}-G 47\right)$ \\
\hline $\begin{array}{l}\mathrm{TiO}_{2} \\
\text { (anatase) } \\
1,2-\mathrm{Ti} \\
3-6-\mathrm{O}\end{array}$ & $(001)$ & Square & $\begin{array}{ll}\text { (1) } & 3 \\
\text { (2) } & 1 \\
\text { (3) } & 4 \\
\text { (4) } & 5 \\
\text { (5) } & 2 \\
\text { (6) } & 6\end{array}$ & $D G 55-P 4 m m m\left(C_{4 v}^{1}-G 99\right)$ \\
\hline $\begin{array}{l}\mathrm{V}_{2} \mathrm{O}_{5} \\
1-4-\mathrm{V} \\
5-8-\mathrm{O} \\
9-12-\mathrm{O} \\
13,14-\mathrm{O}\end{array}$ & $(001)$ & Rectangular & $\begin{array}{l}\text { (1) } 7,8 \\
\text { (2) } 3,4 \\
\text { (3) } 9,10,11,12,13,14 \\
\text { (4) } 1,2 \\
\text { (5) } 5,6\end{array}$ & $D G 37-P m m m\left(D_{2 h}^{1}-G 47\right)$ \\
\hline $\begin{array}{l}\mathrm{Al}_{2} \mathrm{O}_{3} \\
1-4-\mathrm{Al} \\
5-10-\mathrm{O}\end{array}$ & $(111)$ & Hexagonal & $\begin{array}{l}\text { (1) } 1 \\
\text { (2) } 8,9,10 \\
\text { (3) } 2 \\
\text { (4) } 4 \\
\text { (5) } 5,6,7 \\
\text { (6) } 3\end{array}$ & $D G 66-P \overline{3}\left(C_{3 i}^{1}-G 147\right)$ \\
\hline
\end{tabular}

The slab model is now widely used in Hartree-Fock LCAO calculations of crystalline surfaces [6] and also in DFT calculations $[7,8]$.

The layer group contains a subgroup of $2 D$ translations $T^{(2)}$ with elements $\left(E \mid \mathbf{a}_{n}\right)$ where $\mathbf{a}_{n}=n_{1} \mathbf{a}_{1}+n_{2} \mathbf{a}_{2}$ is an arbitrary translation vector of the $2 D$ (plane) lattice, $\mathbf{a}_{1}$ and $\mathbf{a}_{2}$, are primitive unit cell translation vectors, defining a $2 D$ Bravais lattice.

Let us consider the layer groups for the slab models of the following surfaces: $(001)$ in $\mathrm{MgO}$ crystal $(G=F m \overline{3} m)$, (110) in the rutile structure of $\mathrm{TiO}_{2}\left(\mathrm{P}_{2} / \mathrm{mnm}\right),(001)$ in the anatase structure of $\mathrm{TiO}_{2}\left(I 4_{1} /\right.$ amd $),(001)$ in the $\mathrm{V}_{2} \mathrm{O}_{5}$ crystal (Pmmn), and the (111) plane of $\mathrm{Al}_{2} \mathrm{O}_{3}(P \overline{3})$.

In the slab model, the surface plane has a fixed orientation relative to the bulk symmetry group elements. All the atoms of the bulk primitive unit cell are distributed within one or several planes with the same $2 D$ translation symmetry. These planes of atoms form a layer which is by definition stoichiometric. Of practical importance for surface studies using slab models is the dependence of the calculated properties on the number of layers in the slab. The point symmetry group of a slab generally depends on the number of layers included.
In Table 1, the distribution of the bulk structure primitive unit cell atoms over the atomic planes in one-layer slab models is given. For the $\mathrm{MgO}$ crystal one may use not only the bulk primitive unit cell consisting of two atoms, but also the conventional cell consisting of an 8-atomic cubic cell with

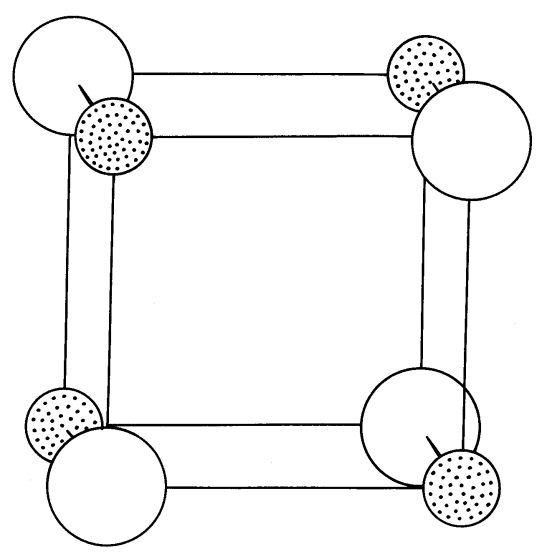

Figure 1. Unit cell of an $\mathrm{MgO}(001)$ surface slab derived from the conventional unit cell of the $3 D$ lattice. Gray spheres: $\mathrm{Mg}$, white spheres: O. 

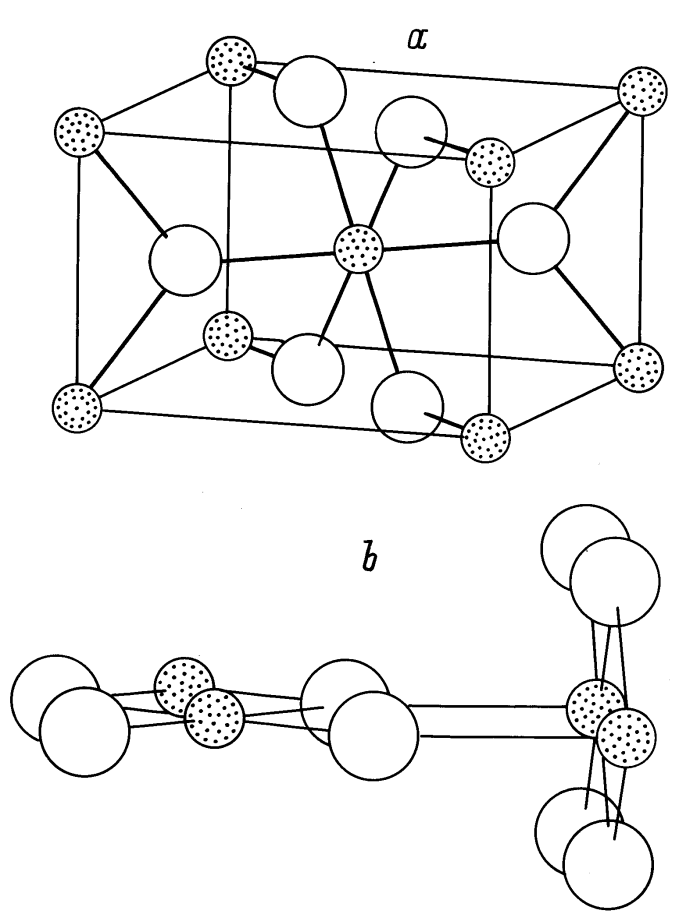

Figure 2. Conventional $3 D$ rutile unit cell $(a)$ and two unit cells of a rutile (110) surface slab derived from the primitive unit cell of the $3 D$ lattice $(b)$. Gray spheres: Ti, white spheres: $\mathrm{O}$.
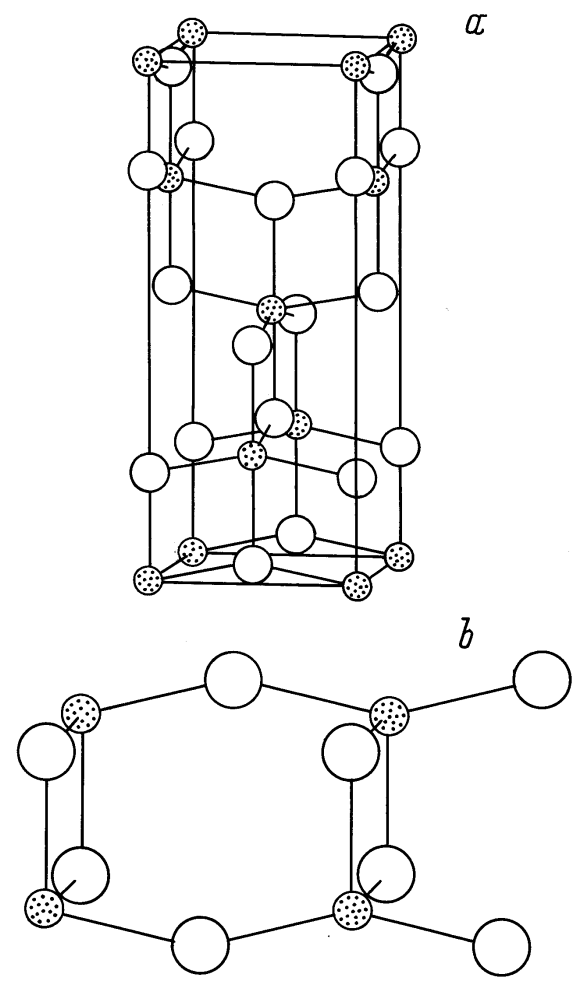

Figure 3. Conventional $3 D$ anatase unit cell $(a)$ and two unit cells of an anatase (001) surface slab derived from the primitive unit cell of the $3 D$ lattice $(b)$. Gray spheres: Ti, white spheres: O. two planes of atoms (Fig. 1). A rutile (110) surface layer consists of three planes of atoms (Fig. 2,a); when choosing the one-layer slab model one takes the following order of planes: oxygen plane, mixed oxygen-titanium plane, oxygen plane (Fig. 2,b). Such a layer choice ensures the zero dipole moment in the slab [6]. For the anatase (001) surface, the six atoms of the primitive bulk unit cell are distributed over six different planes (Fig. 3, $a$ ); so that one layer consists of six atomic planes (Fig. 3, $b$ ). The representative atoms are labeled in Table 1. In the case of the (001) surface of $\mathrm{V}_{2} \mathrm{O}_{5}$ crystal (Fig. 4, $a$ ), the layer consists of five atomic planes (Fig. 4, $b$ and Table 1). In the vanadia bulk structure there are three types of non-equivalent oxygen atoms with different coordination. In the one-layer slab model, these atoms appear equally distributed over three planes of atoms. The primitive bulk unit cell of corundum $\mathrm{Al}_{2} \mathrm{O}_{3}$ (rhombohedral lattice) is shown in Fig. 5, $a$. This unit cell contains two formula units. If the (111) surface is considered, the ten atoms are distributed over six different atomic planes as shown in Fig. 5, $b$ and Table 1. Alternatively, it is also possible to choose the hexagonal unit cell which contains six formula units. The same surface is then labeled (0001) and the 30 atoms of the bulk unit cell are distributed over 12 atomic planes. The slab model is, in fact, an infinite model of a surface since $2 D$ periodicity of the system is maintained. For the computational schemes based on the use of plane waves, the $3 D$ periodicity is restored in so called multislab models considering an infinite number of identical slabs regularly spaced along the normal to the surface (a typical separation is 10 a.u.) [6].

The most common application of the slab model is the study of regular adsorption phenomena. Both the monolayer of the adsorbed species (with the same $2 D$ periodicity as the slab) and lower coverages can be studied in the slab model. In the latter case, the $2 D$ supercell approach is used: the two translation vectors $\mathbf{a}_{1}, \mathbf{a}_{2}$ defining the plane lattice are

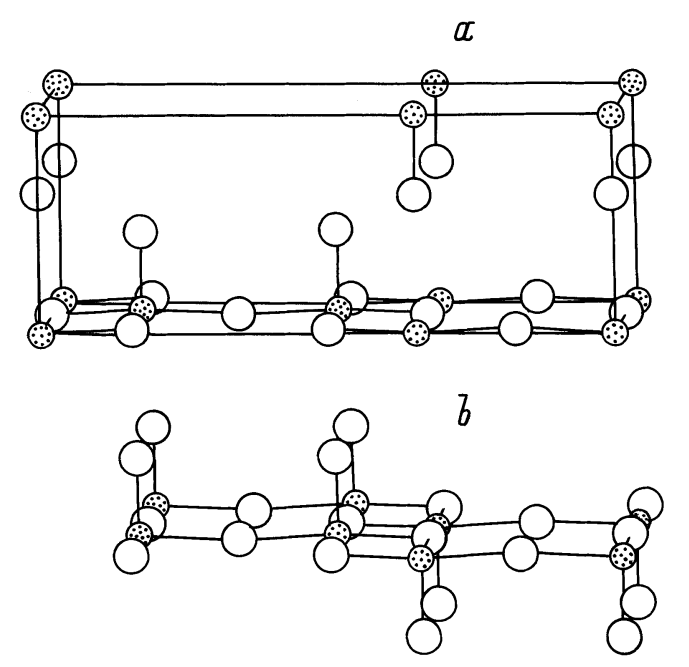

Figure 4. Conventional $3 D \mathrm{~V}_{2} \mathrm{O}_{5}$ unit cell $(a)$ and two unit cells of a $\mathrm{V}_{2} \mathrm{O}_{5}(001)$ surface slab derived from the primitive unit cell of the $3 D$ lattice $(b)$. Gray spheres: V, white spheres: O. 

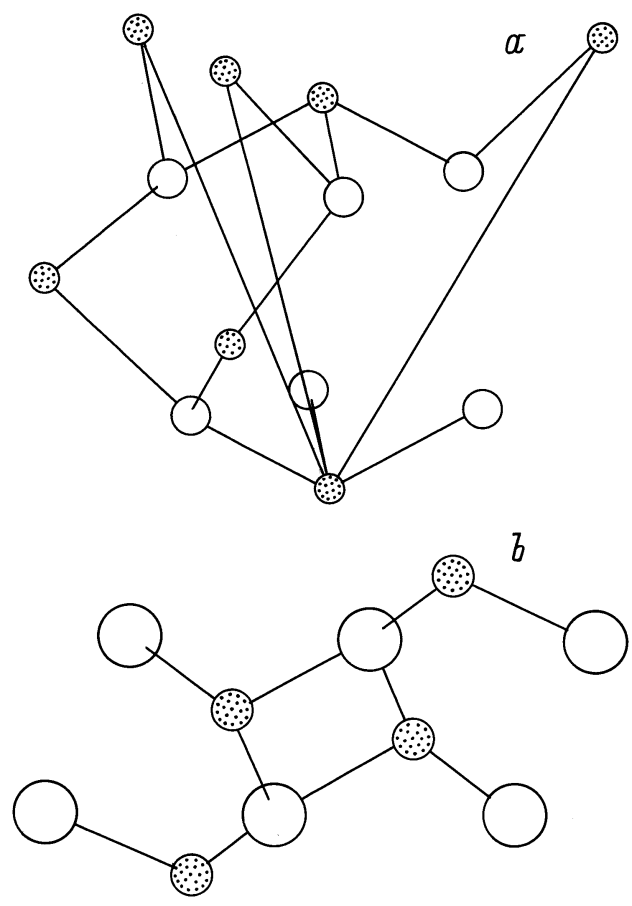

Figure 5. Rhombohedral $3 \mathrm{D} \mathrm{Al}_{2} \mathrm{O}_{3}$ unit cell $(a)$ and unit cell of a $\mathrm{Al}_{2} \mathrm{O}_{3}$ (111) surface slab derived from the primitive unit cell of the $3 D$ lattice $(b)$. Gray spheres: Al, white spheres: O.

transformed with the integer matrix I:

$$
\mathbf{A}_{j}=\sum_{i=1}^{2} l_{j i} \mathbf{a}_{i}, \quad \operatorname{det} \mathbf{l}=L>1 .
$$

The $2 D$ supercell size is defined by the number of primitive unit cells $L$ in the $2 D$ supercell. The transformation (1) is called symmetric, if the point symmetry of the supercell $2 D$ lattice is the same as for the original $2 D$ lattice [9]. The slab-supercell approach introduces a new $2 D$ periodic system and can be treated by the same band theory tools, as the slab itself. The supercell model is now popular in $3 D$ periodic crystals when local defects are studied $[10,11]$. There are no principal differences in the supercell use in $2 D$ and $3 D$ periodic systems. The transformation (1) in the direct lattice generates the transformation of the reciprocal lattice:

$$
\mathbf{b}_{j}=\sum_{i=1}^{2}\left(\mathbf{l}^{-1}\right)_{i j} \mathbf{B}_{i}
$$

where $\mathbf{B}_{i}, \mathbf{b}_{j}$ are the translation vectors of the original and transformed reciprocal lattices, respectively. The new $2 D$ Brillouin zone (BZ) turns out to be $L$ times smaller, so that $L$ points in the initial $\mathrm{BZ}$ coincide:

$$
\mathbf{K}_{l}^{(\mathbf{k})}=\mathbf{k}+\sum_{j=1}^{2} q_{l j} \mathbf{b}_{j}
$$

where $q_{l j}$ are integers and $\mathbf{k}$ is an arbitrary vector in the small BZ.
Thus, in the slab-supercell model the folding of BZ is used, corresponding to a $2 D$ direct lattice, but the system in consideration remains infinite. The adsorbed species in this model are repeated periodically, their distance and interaction with each other depends on the supercell size. Some examples of the band structure for the slab-supercell model are considered, for example in [6].

In surface simulations, other models are also used that are based on the consideration of finite systems.

\section{Finite Models of Surfaces}

2.1. Cyclic clusters. The cyclic cluster (CC) model of surfaces [12-20] is connected with the slab-supercell approach, but there is a difference due to the different introduction of cyclic boundary conditions (CBC). In the latter approach, these conditions are, in fact, introduced for a very large system, e. g., the main region of the $2 D$ periodic plane, so that the band theory methods are used in slab calculations. In the former, the $\mathrm{CBC}$ are introduced for the cluster itself, so that the model turns out to be finite. The convergence of the results to the slab values must be investigated by increasing the $\mathrm{CC}$. But the advantages of the $\mathrm{CC}$ approach are evident:

a) the spurious boundary effects of molecular clusters (discussed in section 2.2) are absent;

b) the periodicity of the adsorbed species or the defect is excluded because the $\mathrm{CC}$ is a finite system;

c) there exists a one-to-one symmetry correspondence between the electronic states of the $2 D \mathrm{CC}$ and the band states of the corresponding slab model.

Let us consider in more detail this correspondence. The $\mathrm{CC}$ model is based on use of the transformation (1) in the $2 D$ direct lattice. However, the $\mathrm{CBC}$ are introduced with the translation vectors $\mathbf{A}_{j}(j=1,2)$ : for oneelectron Bloch function $\Psi_{n \mathbf{k}}(\mathbf{r})$ it is assumed that the relation $\Psi_{n \mathbf{k}}(\mathbf{r})=\Psi_{n \mathbf{k}}\left(\mathbf{r}+\mathbf{A}_{j}\right)=\exp \left(+i \mathbf{k} \mathbf{A}_{j}\right) \Psi_{n \mathbf{k}}(\mathbf{r})$ is fulfilled. Therefore the introduction of CBC defines those irreducible representations $\mathbf{K}$ of the $2 D$ translation group for which

$$
\exp \left(+i \mathbf{K}^{(0)} \mathbf{A}_{j}\right)=1, \quad j=1,2,
$$

i.e. those $\mathbf{K}^{(0)}$ which satisfy Eq. (3) for $\mathbf{k}=0$. Using (1), (4) and expressing $\mathbf{K}^{(0)}=\sum_{i=1}^{2} K_{i}^{(0)} \mathbf{B}_{i}$, one obtains

$$
\begin{aligned}
\sum_{i=1}^{2} \sum_{i^{\prime}=1}^{2} K^{(0)}{ }_{i} \mathbf{l}_{j i^{\prime}}\left(\mathbf{a}_{i^{\prime}} \mathbf{B}_{i}\right) & =\sum_{i=1}^{2} \sum_{i^{\prime}=1}^{2} K^{0}{ }_{i} \mathbf{l}_{j i^{\prime}} 2 \pi \delta_{i, i^{\prime}} \\
& =2 \pi \sum_{i=1}^{2} K^{(0)}{ }_{i} \mathbf{l}_{j i}, \quad j=1,2 .
\end{aligned}
$$

To satisfy (4) the $2 D$ wave vector components are defined by relation

$$
\sum_{i=1}^{2} k_{i} \mathbf{l}_{j i}=m_{j}, \quad j=1,2,
$$

where $m_{j}$ are integers. 


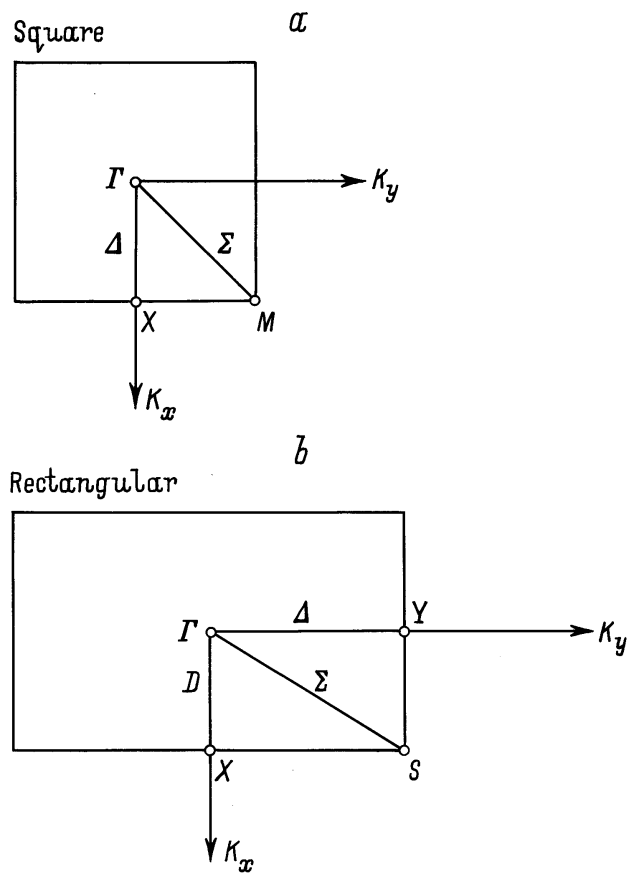

Figure 6. Brillouin zones of square $(a)$ and rectangular $(b)$ 2D lattices.

Eq. (6) is always satisfied for the $\Gamma$ point of $\mathrm{BZ}(\mathbf{K}=0)$, so that the $\mathrm{CC}$ reproduces the states at the $\Gamma$ point of the $2 D \mathrm{BZ}$ independently of its choice. However, for $(\mathbf{K} \neq 0)$ points of a given $2 D$ lattice the set of the electronic states reproduced in the $\mathrm{CC}$ model depends on the choice of the I matrix in (1).

Let us consider as examples two plane lattices, square $\left(\mathrm{MgO}(100)\right.$ and anatase $\mathrm{TiO}_{2}(001)$ surfaces $)$, and rectangular $\left(\mathrm{TiO}_{2}\right.$ rutile (110) and $\mathrm{V}_{2} \mathrm{O}_{5}(001)$ surfaces).

The corresponding $2 D \mathrm{BZ}$ are given in Fig. 6. Let us choose $2 D \mathrm{CC}$ for square lattice in such a way that $C_{4 v}$ point symmetry is not broken. The smallest CC corresponds to

$$
\mathbf{l}=\left(\begin{array}{cc}
1 & -1 \\
1 & 1
\end{array}\right), \quad L=2,
$$

so that Eq. (6) is satisfied for $\Gamma(0,0)$ and $M\left(\frac{1}{2} \frac{1}{2}\right)$ points of $2 D \mathrm{BZ}$. The corresponding point groups of these two $\mathbf{k}$ points are the same, i.e. $\left(C_{4 v}\right)$. The $2 D$ unit cell is two times larger than the primitive one. For transformation (1) with matrix

$$
\mathbf{I}=\left(\begin{array}{ll}
2 & 0 \\
0 & 2
\end{array}\right), \quad L=4
$$

the corresponding $\mathrm{CC}$ reproduces the states at $\Gamma, M$ and $X$ (2 rays) $\mathbf{k}$ vector stars. Further increase of the $\mathrm{CC}$ allows to reproduce not only symmetry points of $2 \mathrm{D} \mathrm{BZ}$ but also points from the symmetry directions. In particular, for

$$
\mathbf{l}=\left(\begin{array}{ll}
3 & 0 \\
0 & 3
\end{array}\right), \quad L=9
$$

the following $\mathbf{k}$ points satisfy (6): $\quad \Gamma(0,0)$, $4 \Delta\left(\frac{1}{3} 0,-\frac{1}{3} 0,0 \frac{1}{3}, 0-\frac{1}{3}\right), 4 \Sigma\left(\frac{1}{3} \frac{2}{3}, \frac{2}{3} \frac{1}{3}, \frac{1}{3} \frac{1}{3}, \frac{2}{3}, \frac{2}{3}\right)$. When the $\mathbf{k}$ points of $2 D$ BZ, satisfying Eq. (6), are found, the induced representations (IR) of $D G$ space groups have to be used to find the symmetry of $\mathrm{CC}$ electron states. To construct the IR table of a given $D G$, the table of the IR for the corresponding triperiodic group may be used [5]. In particular, to obtain the table of IR for DG61 (symmetry group of cyclic clusters of $\mathrm{MgO}(001)$ surface) the table of IR is for the related space group $G 123\left(D_{4 h}^{1}\right)$ has to be used. This table gives the connection between irreducible representations of site symmetry point groups of $D G 61$ and those of the space group itself. For the symmetry directions $\Delta, \Sigma, Y$, the compatibility relations have to be used.

For the rectangular lattice, transformation matrix (7) leads to a CC reproducing the $\Gamma(0,0)$ and $S\left(\frac{1}{2} \frac{1}{2}\right)$ points of the $2 D \mathrm{BZ}$ (Fig. 6). The corresponding $\mathrm{CC}$ for transformation (8) reproduces states at $\Gamma(0,0), X\left(\frac{1}{2} 0\right), Y\left(0 \frac{1}{2}\right)$, and $S\left(\frac{1}{2} \frac{1}{2}\right)$. Further increase of the $\mathrm{CC}$ using transformation (9) allows to reproduce the symmetry point $\Gamma(0,0)$ and symmetry directions $D\left(\frac{1}{3} 0,-\frac{1}{3} 0\right), \Delta\left(0 \frac{1}{3}, 0-\frac{1}{3}\right)$, and $\Sigma\left(\frac{1}{3} \frac{1}{3},-\frac{1}{3} \frac{1}{3}, \frac{1}{3}-\frac{1}{3},-\frac{1}{3}-\frac{1}{3}\right)$. This consideration clarifies the symmetry connection between the CC model and supercellslab models.

The scheme of $\mathrm{CC}$ choice in consideration was applied [21] to calculate the adsorption geometry for organic adsorbates on a rutile (110) surface. The INDO parametrization scheme was modified to reproduce experimental results for geometries of carbon and nitrogen containing molecules. The CC representing the rutile (110) surface [21] consists of 120 atoms. It includes two layers of $5 \times 22 D$ unit cells with 6 atoms in each $2 D$ unit cell.

2.2. Molecular clusters. The molecular cluster (MC) model is extremely popular in surface simulations because of its simplicity, flexibility and the possibility to use the standard techniques of molecular quantum chemistry [4,22-25]. In the MC approach, a crystal with a surface is modeled by a finite (molecular) system consisting of the atoms on the surface and of some atomic planes nearest to it. The $2 D$ periodicity of the surface is not taken into account, so that the symmetry of such a model is described by one of the crystallographic point groups. In adsorption studies, the adsorbed species are included in the cluster-adsorbate systems.

A MC is cut out from the slab to simulate a portion of the surface, so that spurious effects arise, related to the limited cluster size and to the presence of the boundary. To eliminate these effects the dangling bonds are saturated either by hydrogen atoms or pseudoatoms, or the whole cluster is embedded in the external field simulating the influence of the bulk crystal $[24,26,27]$.

In the MC model, the results critically depend on the choice of the cluster size, stoichiometry and shape. Nevertheless, the MC model can provide useful information if the cluster is properly chosen and the influence of its size is investigated.

In the following, we suggest a number of general rules for the generation of MC suitable for surface simulations based on the connection between the MC and the slab model. 


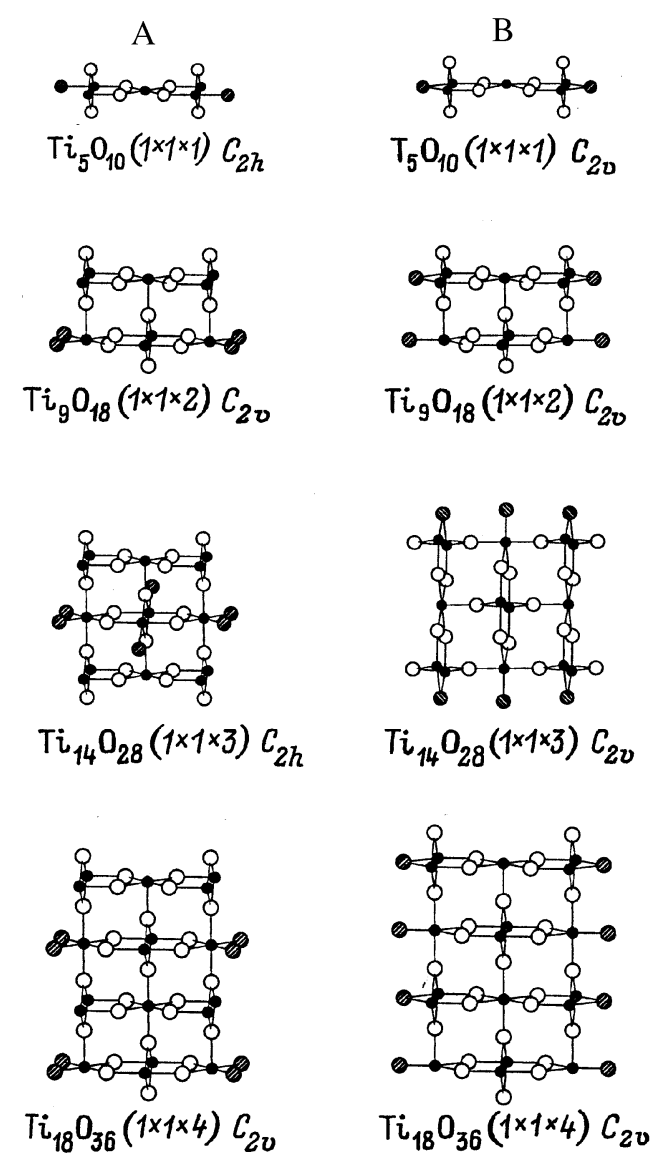

Figure 7. Rutile (110) surface of size $1 \times 1$ simulated with 1-4layer clusters of types $A$ and $B$. Black spheres: Ti, white spheres: $\mathrm{O}$, shaded spheres: additional $\mathrm{O}$ added to achieve stoichiometry.
We are aware of the fact that in previous studies these rules have been intuitively applied, in most cases without explicitly considering the relationship between periodic and molecular models. Nevertheless, we believe that it might be useful for future surface studies to consider these rules in order to minimize artefacts of the models:

a) the clusters correctly reflect the stoichiometry of the bulk crystal and are electro-neutral;

b) atoms on the crystal surface should be equivalent on the cluster surface except for corners and edges;

c) the average coordination of all cluster atoms is as close as possible to that on the crystal surface;

d) as many symmetry elements of the crystal surface as possible are retained in the cluster;

e) an extension to rule a) is that each layer of the cluster should have the stoichiometry of the crystal.

In fact, such principles of the MC choice ensure the simulation of the crystal surface as close as possible and are directly connected with the $2 D$ periodical slab model. By using $2 D$ unit cells as basic units of model clusters, it is possible to increase the cluster size in a systematical way and thereby to study the convergence behavior of calculated surface properties.

As examples of MC model applications to surface studies using some of the above mentioned rules there may be considered calculations of $\mathrm{MgO}, \mathrm{TiO}_{2}$ (rutile, anatase structures), and $\mathrm{Cr}_{2} \mathrm{O}_{3}$ (corundum structure) surfaces and adsorption of small molecules on them [23-25,28-37]. But it is also possible to find examples in the literature where these rules have not been applied [38,39] and in part unphysical results were obtained.
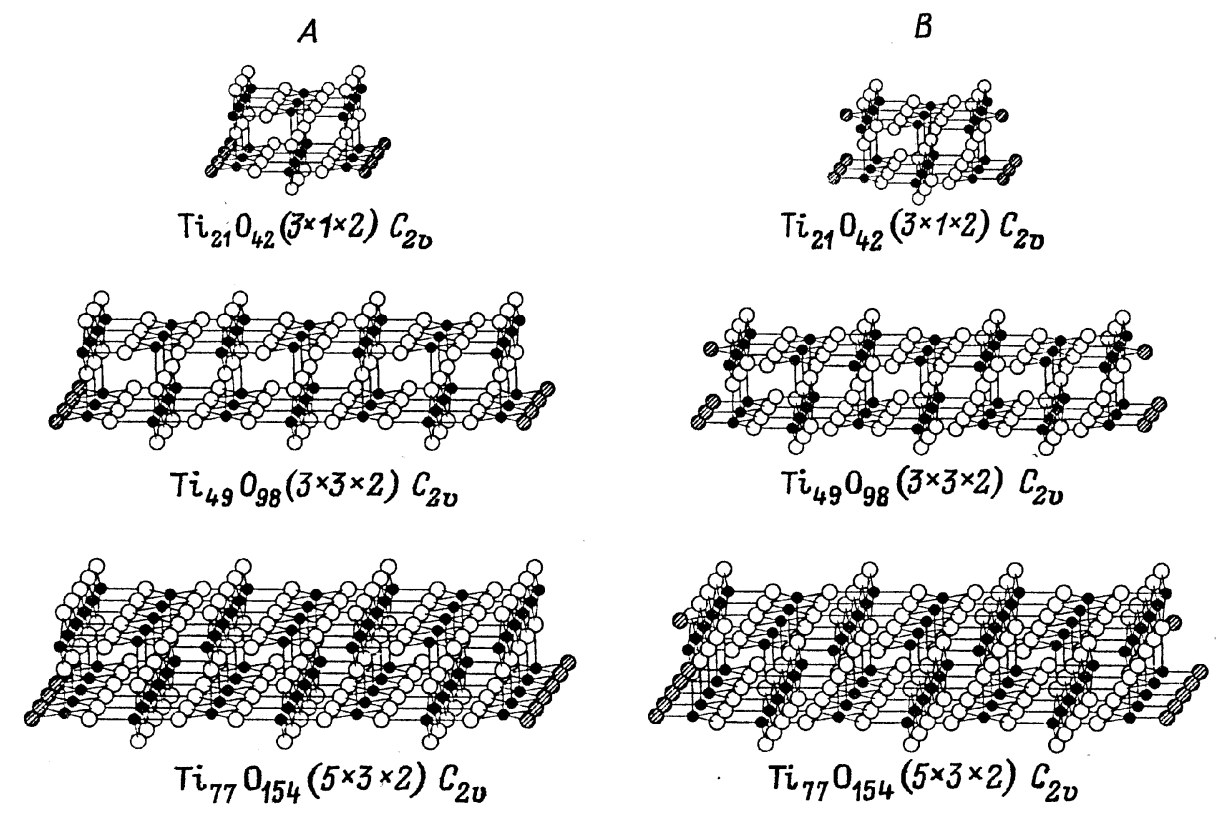

Figure 8. Rutile (110) surfaces of size $3 \times 1,3 \times 3$, and $5 \times 3$ simulated with two-layer clusters of types $A$ and $B$. Black spheres: Ti, white spheres: $\mathrm{O}$, shaded spheres: additional $\mathrm{O}$ added to achieve stoichiometry. 
Table 2. MSINDO Adsorption Energies $(\mathrm{kJ} / \mathrm{mol})$ for Molecular and Dissociative Water Adsorption on Rutile (110) Calculated with Relaxation. The Clusters Correspond to Those Presented in Figs. 7 and 8

\begin{tabular}{l|c|c|c|c}
\hline \multirow{2}{*}{ Cluster } & \multicolumn{2}{|c|}{ Type $A$} & \multicolumn{2}{c}{ Type $B$} \\
\cline { 2 - 5 } & molecular & dissociative & molecular & dissociative \\
\hline $\mathrm{Ti}_{5} \mathrm{O}_{10}$ & 51 & 39 & 70 & 201 \\
$\mathrm{Ti}_{9} \mathrm{O}_{18}$ & 139 & -24 & 94 & 107 \\
$\mathrm{Ti}_{14} \mathrm{O}_{28}$ & 66 & 8 & 92 & 143 \\
$\mathrm{Ti}_{18} \mathrm{O}_{36}$ & 157 & 192 & 129 & 162 \\
$\mathrm{Ti}_{49} \mathrm{O}_{98}$ & 120 & 151 & 119 & 157
\end{tabular}

In $\mathrm{MgO}$ and $\mathrm{NaCl}$ crystals, the $\mathrm{MC}$ were chosen in such a way [33] that they simulate the supercell of the surface and include several layers of the bulk crystal. For these crystals, the stoichiometry of the MC chosen was ensured by such a choice.

For more complicated systems, e.g. $\mathrm{TiO}_{2}$ structures, the stoichiometry of the MC is only ensured when several additional oxygen atoms are added. There are two principle ways to add these additional atoms. They can be placed at positions of the regular surface around the cluster. This way is denoted as type $A$ as shown in Figs. 7 and 8 . In some cases, this procedure leads to clusters that do not correspond to rules d) and e). Another possibility is to place the additional atoms for saturation not at regular positions of the crystalline lattice so that rules d) and e) are fulfilled. Examples of this second type $B$ of cluster generation are presented in Figs. 7 and 8. If all layers of a multi-layer cluster have the same stoichiometry, artificial polarization is reduced which can affect the calculated surface properties. This effect is investigated in the next section.

2.3. Numerical results. In order to study the effect of the distribution of saturation atoms on calculated properties of crystalline surfaces, we have selected the rutile (110) surface for new MSINDO calculations. A large number of theoretical studies have been performed using the $\mathrm{MC}$ model for the simulation of this surface [28,30-32,34-39]. We used the semi-empirical SCF MO method MSINDO [40], the successor of the SINDO1 method that was successfully applied in studies of metal oxide surfaces [23-25,28,29]. Water was adsorbed in molecular and dissociated form on the clusters $\mathrm{Ti}_{5} \mathrm{O}_{10}, \mathrm{Ti}_{9} \mathrm{O}_{18}, \mathrm{Ti}_{14} \mathrm{O}_{28}$, and $\mathrm{Ti}_{18} \mathrm{O}_{36}$ (Fig. 7) chosen in accordance with the rules suggested here. Nevertheless, even if these rules are applied, several different types of clusters may be constructed. Two types of possible cluster models were used. In type $A$ clusters, already used previously [28], the additional oxygen atoms needed to ensure total stoichiometry (shaded in Fig. 7) were placed at regular lattice positions. In type $B$ clusters, considered by us for the first time, the additional oxygen atoms were placed at non-lattice positions in order to maximize symmetry of the clusters and to reduce polarization as discussed in the previous section. The adsorption energies calculated with these small cluster models are presented in Table 2.
The geometries of the clusters were optimized within the symmetry of the rutile structure. In model type $B$, there are one or two more degrees of freedom for those oxygen atoms that are in non-lattice positions. For the cluster-water systems, the Cartesian coordinates of all water atoms and of the two surface atoms close to the oxygen atom and the hydrogen atom of water are optimized. The adsorption energy is calculated as the difference of the total energies of the isolated systems and the cluster- $\mathrm{H}_{2} \mathrm{O}$ system. Therefore, positive values indicate a stabilization.

A comparison is made with the results from calculations on a much larger cluster, $\mathrm{Ti}_{49} \mathrm{O}_{98}$ (Fig. 8), which is a better representation of the surface. From Table 2 it can be seen that the different distribution of additional oxygen atoms in cluster types $A$ and $B$ has a substantial influence on the calculated adsorption energies. The variation of adsorption energy with an increasing number of layers is significantly reduced if model type $B$ is considered. Even for rather small systems the adsorption energies are relatively close to that of the largest cluster $\mathrm{Ti}_{49} \mathrm{O}_{98}$. The most important difference between the two types of models is that only for type $B$ the dissociative adsorption is always more stable that the molecular form. The relative stability of the two forms of water on the rutile (110) surface is still a matter of debate [36]. We do not want to engage ourselves here in this controversy, and focus on the convergence of results obtained for clusters with increasing size.

The comparison of types $A$ and $B$ of the largest model $\mathrm{Ti}_{49} \mathrm{O}_{98}$ show that the influence of the additional atoms is negligible due to their large distance to the adsorption position. This indicates that in this case it is rather the description of the local environment near the adsorption site than the effect of a global polarization of the cluster that is responsible for the differences observed for the smaller clusters.

\section{Conclusion}

The consideration of symmetry in models of crystalline surfaces (infinite-slab model and finite MC and CC models) allows to give general criteria for the choice of the MC used to simulate the crystalline surface. Based on the distribution of atoms of the bulk primitive unit cell over atomic planes for a given surface orientation, stoichiometric and electro-neutral clusters are chosen. As many point symmetry elements of the slab model as possible are retained in the cluster. Surface atoms of the cluster have equivalent surrounding, if they are equivalent in the slab or the $\mathrm{CC}$, except for corners and edges. If the cluster contains several layers, each of them must have the same stoichiometry.

The numerical results for the adsorption behavior of rutile (110) towards water with different clusters demonstrate that convergence to the methodological limit is significantly improved if the suggested criteria are taken into account. Since the underlying considerations are of general nature, they can be applied also to cluster models of surfaces of other systems. 
One of the authors (R.A. E.) thanks Alexander von Humboldt-Stiftung for a Humboldt Award. This work was partially supported by Deutsche Forschungsgemeinschaft.

The structure drawings were performed with SCHAKAL97.

\section{References}

[1] G.A. Somorjai. Introduction to surface chemistry and catalysis. Wiley, N. Y. (1994).

[2] V.E. Henrich, P.A. Cox. The Surface Science of Metal Oxides. Cambridge University Press, Cambridge (1994).

[3] J. Sauer. Chem. Rev. 89, 199 (1989).

[4] P. Deák. Phys. Stat. Sol. (b) 217, 9 (2000).

[5] R.A. Evarestov, V.P. Smirnov. Site Symmetry in Crystals: Theory and Applications. 2nd ed. Springer, Berlin-HeidelbergN. Y. (1997).

[6] C. Pisani. In: Quantum-Mechanical Ab-Initio Calculation of the Properties of Crystalline Materials / Ed. C. Pisani Lecture Notes in Chem. Springer, Berlin-Heidelberg-N. Y. (1996).

[7] T. Bredow, G. Pacchioni. Surf. Sci. 426, 106 (1999).

[8] P.J.D. Lindan, J. Muscat, S. Bates, N.M. Harrison, M. Gillan. Farad. Discuss. 106, 135 (1997).

[9] R.A. Evarestov, V.P. Smirnov. J. Phys. C: Cond. Matter 9, 3023 (1997).

[10] A. Lichanot, C. Zarrieu, C. Zicovich-Wilson, C. Roetti, R. Orlando, R. Dovesi. J. Phys. Chem. Sol. 59, 1119 (1998).

[11] W.C. Mackrodt, E.A. Williamson. Phil. Mag. B77, 1077 (1998).

[12] A.J. Bennet, B. Mc Carrol, R.P. Messmer. Surf. Sci. 24, 191 (1971); Phys. Rev. B3, 1397 (1971).

[13] A.M. Dobrotvorskii, R.A. Evarestov. Phys. Stat. Sol. (b) 66, 83 (1974).

[14] A. Zunger. J. Chem. Phys. 62, 1861 (1975); Ibid. 63, 1713 (1975).

[15] R.A. Evarestov, M.I. Petrashen, E.M. Ledovskaya. Phys. Stat. Sol. (b) 76, 377 (1976).

[16] P. Deák. Acta Phys. Acad. Sci. Hung. 50, 247 (1981).

[17] R.A. Evarestov, A.V. Leko, V.P. Smirnov. Phys. Stat. Sol. (b) 128, 275 (1985).

[18] J. Stewart. J. Comput. Chem. 19, 168 (1998).

[19] J. Noga, P. Bańacký, S. Biskupič, R. Boča, P. Pelikan, M. Svrček, A. Zajac. J. Comput. Chem. 20, 253 (1999).

[20] T. Bredow, R.A. Evarestov. Phys. Stat. Sol. (b) 220, R5 (2000).

[21] P. Persson, A. Stashans, R. Bergström, S. Lunell. Int. J. Quantum Chem. 70, 1055 (1998).

[22] C. Pisani. J. Mol. Struc. (Theochem) 463, 125 (1999).

[23] K. Jug. Int. J. Quantum. Chem. 58, 283 (1996).

[24] T. Bredow, G. Geudtner, K. Jug. J. Chem. Phys. 105, 6395 (1996).

[25] V.A. Tikhomirov, G. Geudtner, K. Jug. J. Phys. Chem. B101, 10398 (1997).

[26] U. Gutdeutsch, U. Birkenheuer, S. Krüger, N. Rösch. J. Chem. Phys. 106, 6020 (1997).

[27] C. Pisani, S. Casassa, F. Corà. Comput. Phys. Commun. 82, 139 (1994).

[28] T. Bredow, K. Jug. Surf. Sci. 327, 398 (1995).

[29] T. Bredow. Surf. Sci. 401, 82 (1998).

[30] A. Hagfeldt, H. Siegbahn, S.-E. Lindquist, S. Lunell. Int. J. Quantum Chem. 44, 477 (1992).
[31] G. Pacchioni, A.M. Ferrari, P.S. Bagus. Surf. Sci. 350, 159 (1996).

[32] P. Reinhardt, M. Causà, C.M. Marian, B.A. Heß. Phys. Rev. B 54, 14812 (1996).

[33] K. Jug, G. Geudtner. J. Mol. Cat. A119, 143 (1997).

[34] F. Rittner, R. Fink, B. Boddenberg, V. Staemmler. Phys. Rev. B57, 4160 (1998).

[35] M.A. San Miguel, C.J. Calzado, J.F. Sanz. Int. J. Quantum Chem. 70, 351 (1998).

[36] E.V. Stefanovich, T.N. Truong. Chem. Phys. Lett. 299, 623 (1999).

[37] M. Casarin, C. Maccato, A. Vittadini. Appl. Surf. Sci. 142, 196 (1999).

[38] H. Kobayashi, M. Yamaguchi. Surf. Sci. 214, 466 (1989).

[39] K.D. Schierbaum, W.-X. Xu. Int. J. Quantum Chem. 57, 1121 (1996).

[40] B. Ahlswede, K. Jug. J. Comput. Chem. 20, 563 (1999). 